\title{
Bullöse Arzneimittelreaktion im Bereich subkutaner
} Methotrexat-Injektionen

\author{
Bullous Drug Reaction in Areas of Subcutaneously Applied Methotrexate
}

\section{Zusammenfassung}

Zur Behandlung einer rheumatoiden Arthritis war der hier vorgestellten Patientin Methotrexat verordnet worden, das sie sich seither wöchentlich in einer Dosierung von $10 \mathrm{mg}$ subkutan in die Bauchhaut injiziert hatte. Sechs Monate nach Einleitung dieser Therapie waren erstmals Erytheme und Blasen an der Bauchhaut bemerkt worden, die auch in den folgenden Monaten bei Fortführung der Behandlung ausschließlich im Bereich der Injektionsstellen auftraten. Die Pathogenese dieser Arzneimittelreaktion ist unklar und lässt sich weder durch allergische noch durch toxische Mechanismen erklären.
Abstract

A once weekly subcutaneous injection of $10 \mathrm{mg}$ methotrexate was prescribed for a patient who suffered from rheumatoid arthritis. Six months after initiation of therapy, the patient noticed erythema and blisters at the abdominal skin for the first time. These symptoms were exclusively observed at the injection sites during the following months. Pathogenesis of the described drug reaction remains uncertain. There is every reason to believe that pathogenesis is neither of allergic nor of toxic nature.

\section{Einleitung}

Obwohl seit Jahrzehnten bekannt, gilt Methotrexat auch heute noch als etablierte Substanz bei der Behandlung pathogenetisch unterschiedlicher Erkrankungen in der Onkologie, der Rheumatologie und der Dermatologie. Dabei wird Methotrexat in der Onkologie meist in Form einer Polychemotherapie bei Leukämien und Lymphomen, gynäkologischen Neoplasien, kleinzelligen Bronchialkarzinomen und Osteosarkomen eingesetzt [1]. $\mathrm{Zu}$ den typischen rheumatologischen Indikationen einer Methotrexat-Therapie zählen die rheumatoide Arthritits und die Psoriasis arthropathica [2]. In der Dermatologie wird Methotrexat hauptsächlich bei der ausgedehnten Manifestation der Psoriasis vulgaris, bei der psoriatischen Erythrodermie und bei der Psoriasis pustulosa Zumbusch verordnet [3,4]. Darüber hinaus hat sich Methotrexat auch bei einer Vielzahl weiterer Dermatosen als wirksame Therapieoption bewährt, z. B. bei den blasenbildenden
Dermatosen, Vaskulitiden, Kollagenosen und kutanen T-ZellLymphomen [5]. Die Nebenwirkungen einer Methotrexat-Therapie sind vielfältig. Im Vordergrund stehen Einschränkungen des Allgemeinbefindens, wie z. B. Zephalgien, Fieber und Gewichtsverlust sowie die ebenfalls häufig vorkommenden gastrointestinalen Beschwerden, zu denen Übelkeit und Erbrechen, Diarrhöen und Ulcera ventriculi gezählt werden $[6,7]$. Bei einer hochdosierten oder langfristig durchgeführten Methotrexat-Therapie muss bei etwa $30 \%$ der behandelten Patienten mit hepatotoxischen Reaktionen gerechnet werden [6]. Das Spektrum der hepatogenen Nebenwirkungen umfasst Transaminasenerhöhungen ohne klinische Symptome, die akute Hepatitis, die Fibrose und die Zirrhose. Neben der kumulativen Gesamtdosis begünstigen verschiedene Risikofaktoren, wie z. B. ein vorbestehender Leberschaden und ein Alkohol- oder Medikamentenabusus, die hepatogene Toxizität $[8,9]$. Weitere Nebenwirkungen einer Methotrexat-Therapie, die auch dosisunabhängig beobachtet werden, sind 
Störungen der Hämatopoese, die einzelne Zellreihen betreffen oder als Panzytopenie auftreten [10]. Die seltener vorkommende Methotrexat induzierte Pneumonie zeigt typischerweise einen klinisch subakuten Verlauf mit nicht produktivem Husten, Dyspnoe und Fieber. Der radiologische Befund entspricht einer interstitiellen Pneumonie [2,11]. Mit Ausnahme der Alopezie, deren Häufigkeit mit etwa $6 \%$ angegeben wird, sind die kutanen Nebenwirkungen einer Methotrexat-Therapie selten. Das klinische Spektrum der kutanen Nebenwirkungen ist jedoch durch eine Vielzahl sehr unterschiedlicher Dermatosen gekennzeichnet, zu denen Arzneimittelexantheme, anaphylaktische Reaktionen, Ulzera der Haut und Schleimhäute, Reaktivierungen einer Dermatitis solaris, Rheumaknoten und Lymphome bzw. Pseudolymphome gezählt werden [12-16]. Die Pathogenese dieser kutanen Nebenwirkungen ist auf allergische, toxische oder bisher nicht geklärte Mechanismen zurückzuführen. Zu den klinisch und pathogenetisch somit sehr unterschiedlichen Nebenwirkungen einer Methotrexat-Therapie muss auch die in der folgenden Kasuistik beschriebene bullöse Arzneimittelreaktion gezählt werden, die in ihrer klinischen Manifestationsform in der uns zur Verfügung stehenden Literatur bisher nicht beschrieben worden ist.

\section{Kasuistik}

\section{Anamnese}

Bei der jetzt 68-jährigen Patientin war im frühen Erwachsenenalter eine rheumatoide Arthritis aufgetreten, die seither mit Glukokortikoiden oder verschiedenen nichtsteroidalen Antiphlogistika behandelt worden war. Im Juli 2004 erhielt die Patientin erstmals Methotrexat (Lantarel ${ }^{\mathrm{R}}$ FS $10 \mathrm{mg}$ ), das als subkutane Injektionstherapie in einer Dosierung von $10 \mathrm{mg}$ einmal wöchentlich verordnet wurde. Die von der Patientin im Bereich der Bauchhaut selbst vorgenommenen Injektionen wurden zunächst ohne feststellbare Nebenwirkungen vertragen. Die rheumatologische Symptomatik bildete sich vollständig zurück. Sechs Monate nach Einleitung der Behandlung bemerkte die Patientin Juckreiz, Rötungen und Blasen im Bereich der Injektionsstellen. Die Symptome traten jeweils 7-10 Tage nach den Injektionen auf und bildeten sich innerhalb von 3-4 Wochen langsam zurück. Auch bei Fortsetzung der Behandlung zeigten sich die beschriebenen Nebenwirkungen immer nur im Bereich der Injektionsstellen, das übrige Hautorgan und die Schleimhäute blieben erscheinungsfrei. Anaphylaktische Reaktionen wurden auch in den nächsten 10 Monaten nicht bemerkt.

In der weiteren Arzneimittelanamnese der Patientin konnte eine zusätzliche antirheumatische Behandlung mit den nichtsteroidalen Antiphlogistika Acemetacin und Novaminsulfon in einer täglichen Dosierung von $2 \times 60 \mathrm{mg}$ bzw. $3 \times 500 \mathrm{mg}$ erfragt werden. Diese Medikamentation war bereits vor Beginn der Methotrexat-Therapie eingeleitet und dann beibehalten worden. Darüber hinaus erfolgte eine umfangreiche antihypertone Behandlung der Patientin mit Losartan, Hydrochlorothiazid, Bisoprolol und Nitrendipin in den jeweils üblichen Dosierungen.

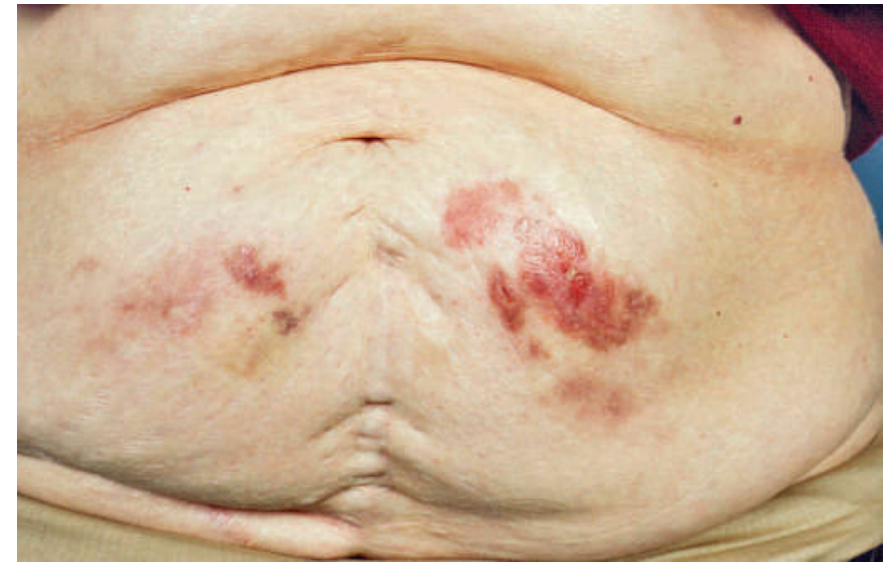

Abb. 1 Infiltrierte Erytheme mit postbullösen Erosionen.

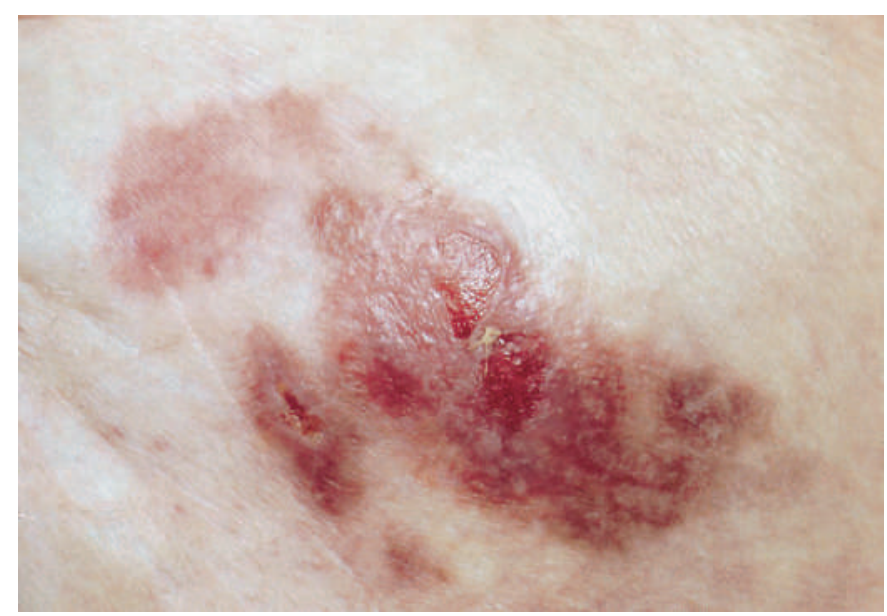

Abb. 2 Detailaufnahme.

\section{Dermatologischer Befund}

An der vorderen Bauchhaut bis mehrfach münzgroße, relativ scharf begrenzte, unterschiedlich infiltrierte Erytheme, teilweise mit umschriebenen postbullösen Erosionen (Abb.1 und 2).

\section{Histopathologischer Befund}

In scharf begrenzten Zonen der Epidermis nekrotische Keratinozyten bei umschriebener Abhebung der Epidermis von der Dermis. Im Randbereich vakuoläre Veränderungen an der Junktion. Oberflächliches Infiltrat aus Lymphozyten. Keine Eosinophile (Abb. 3 und 4).

\section{Direkte Immunfluoreszenz}

Keine Ablagerung von Immunglobulinen oder Komplement.

\section{Histopathologische Begutachtung}

Superfizielle vakuoläre Interface-Dermatitis mit großflächiger Epidermisnekrose. Der Befund entspricht einer schweren bullösen Arzneimittelreaktion, z. B. vom Typ des Stevens-JohnsonSyndroms bzw. der toxischen epidermalen Nekrolyse (PD Dr. W. Weyers, Einsendungslabor für Dermatohistologie, Freiburg). 


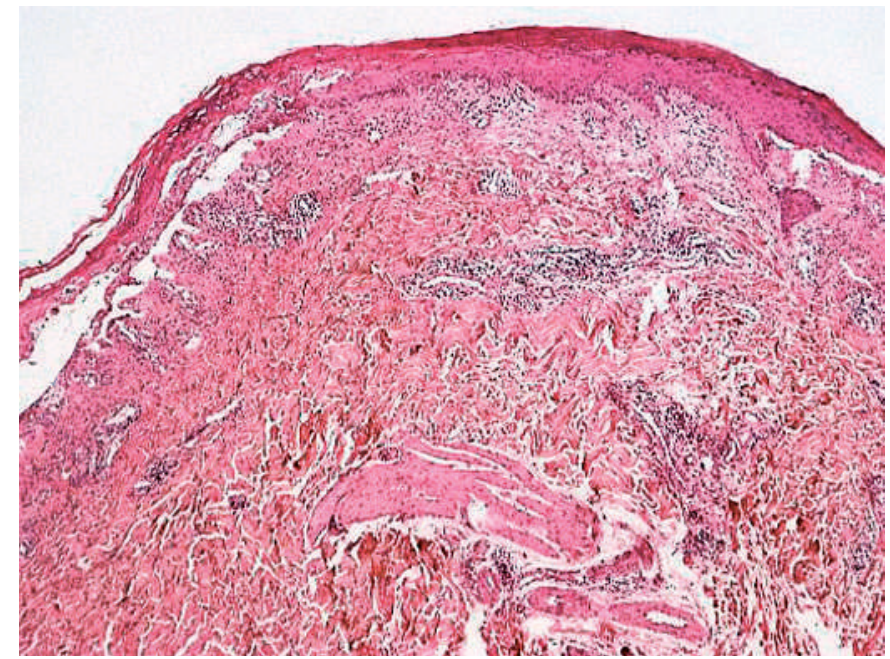

Abb. 3 Superfizielles perivaskuläres Lymphozyteninfiltrat mit subepidermaler Spaltbildung (HE 1:40).

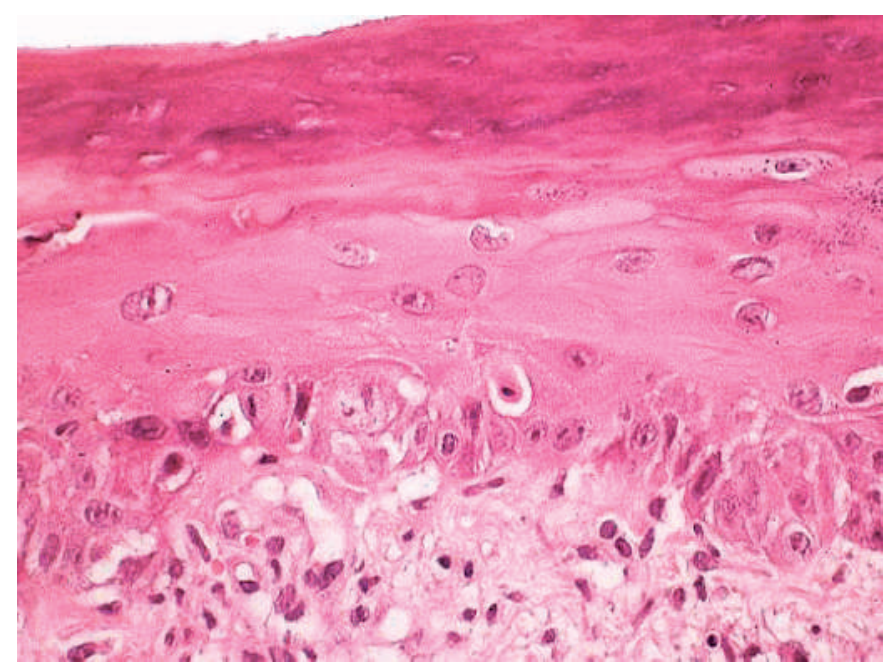

Abb. 4 Nekrotische Keratinozyten und vakuoläre Veränderungen an der Junktionszone (HE 1:400).

\section{Allergologische Testungen}

In Hinblick auf die Anamnese der Patientin wurden bei allen Testverfahren Spätablesungen bis zum 10. Tag nach Applikation vorgenommen. Das Präparat Lantarel ${ }^{\mathrm{R}} \mathrm{FS} 10 \mathrm{mg}$ enthält neben dem Wirkstoff Methotrexat-Dinatrium nur Natriumchlorid, Natriumhydroxid und Wasser für Injektionszwecke. Weitere Zusätze sind nicht enthalten.

Prick- und Scratch-Testungen mit $0,025 \mathrm{mg}$ Methotrexat $/ 1 \mathrm{ml}$ NaCL 0,9\%: Negativ.

Epikutan-Testungen mit 1\% und 3\% Methotrexat in Ungt. emulsificans aquosum $30 \%$ : Negativ.

Offener Epikutan-Test mit Lantarel ${ }^{\mathrm{R}}$ FS $10 \mathrm{mg}$ : Negativ.

Subkutane Injektionen mit 1,3 und 10 mg Methotrexat (Lantarel $^{R}$ FS $10 \mathrm{mg}$ ): Bei 1 und $3 \mathrm{mg}$ Methotrexat keine positive Reaktion; bei $10 \mathrm{mg}$ nach 7 Tagen infiltriertes Erythem, nach 9 Tagen postbullöse Erosionen.

\section{Laborbefunde}

Blut- und Differentialblutbild, Transaminasen, nierenpflichtige Substanzen und Eiweißelektrophorese ohne pathologische Befunde. Allergenspezifischer CAST-Elisa mit Methotrexat $<1 \mathrm{pg} / \mathrm{ml}$ (Referenzbereich $<200 \mathrm{pg} / \mathrm{ml}$ ).

\section{Verlauf}

Nach Abbruch der Methotrexat-Therapie zeigte sich zunächst eine unerwartet verzögerte Rückbildung der Erytheme. Erst 3 Monate nach der letzten Injektion waren die Erytheme unter Hinterlassung ausgeprägter postinflammatorischer Pigmentierungen abgeheilt. Bei erneuter Zunahme der Schmerzsymptomatik wurde der Patientin in Ergänzung zur fortgeführten Behandlung mit Acemetacin und Novaminsulfon zusätzlich Hydroxy-Chloroquin verordnet, ohne dass jedoch die unter Methotrexat erzielte vollständige Beschwerdefreiheit erreicht werden konnte.

\section{Diskussion}

Methotrexat und seine intrazellulär gebildeten, langlebigen Polyglutamat-Derivate zählen als Folsäure-Antagonisten zur Gruppe der Antimetaboliten. Die meisten der bis heute bekannt gewordenen Folsäure-Antagonisten hemmen kompetitiv die Bindung der Folsäure, einem obligaten Nahrungsmittelbestandteil, an die Dihydrofolsäurereduktase. Die daraus resultierende Synthesestörung der Tetrahydrofolsäure, einem essentiellen Lieferanten von $C_{1}$-Bausteinen für den Nuklein- und Aminosäurenstoffwechsel, führt in Abhängigkeit von der Methotrexat-Konzentration im Serum zunächst zu einer Inhibition der DNA-Synthese und bei höheren Konzentrationen auch zu einer solchen der RNA- sowie der gesamten Proteinsynthese [17]. Methotrexat wird nach oraler, intramuskulärer oder subkutaner Applikation rasch resorbiert und zu etwa 50\% an Plasmaproteine gebunden, vor allem an Albumin. Dabei kann Methotrexat durch zahlreiche andere Arzneimittel aus seiner Serumproteinbindung verdrängt werden, z. B. durch Antibiotika, nichtsteroidale Antiphlogistika, Antikonvulsiva oder Antikonzeptiva [18]. Da Methotrexat nur in der freien, nicht an Proteine gebundenen Form pharmakologisch wirksam ist, können die genannten Arzneimittelgruppen neben den therapeutischen Effekten auch die toxischen Nebenwirkungen des Methotrexats steigern. Neben einer Hypoalbuminämie oder medikamentösen Interaktionen sind Störungen der renalen Elimination ein weiterer Mechanismus, der zur Zunahme der Toxizität von Methotrexat führen kann. Nach intravenöser Gabe wird Methotrexat renal in einer dreiphasigen Kinetik chemisch weitgehend unverändert ausgeschieden. Die terminale Phase der Elimination ist bei einer Niereninsuffizienz deutlich verlängert und damit verantwortlich für die toxisch bedingten, hämatologischen und gastroenterologischen Nebenwirkungen [17]. Die Toxizität einer Methotrexat-Behandlung ist somit einerseits auf die substanzspezifischen pharmakologischen Wirkungen eines Antimetaboliten zurückzuführen und andererseits auf verschiedene patienteneigene Faktoren, die unter anderem die Proteinbindung im Serum oder die renale Ausscheidung von Methotrexat beeinflussen. Im Vergleich zu den zahlenmäßig im Vordergrund stehenden internistischen und hier typischerweise to- 
xisch bedingen Komplikationen einer Methotrexat-Behandlung sind die dermatologischen Nebenwirkungen insgesamt selten, zeichnen sich jedoch gleichzeitig durch eine ungewöhnliche Vielzahl klinisch unterschiedlicher Krankheitsbilder aus. Die Pathogenese der dermatologischen Komplikationen ist dabei ebenfalls vielfältig. Neben toxischen und allergischen Mechanismen konnte die Pathogenese einzelner durch Methotrexat ausgelöster Dermatosen bis heute nicht geklärt werden.

\section{Alopezie als häufigste kutane Nebenwirkung}

Die am häufigsten auftretende kutane Nebenwirkung einer Methotrexat-Therapie ist die toxisch bedingte Alopezie, die bei etwa $6 \%$ der Patienten beobachtet werden kann [6]. Im Trichogramm findet sich ein telogenes, bei einer Hochdosistherapie bisweilen auch ein anagenes Effluvium [5]. Als weitere ebenfalls toxisch verursachte Folgen einer Methotrexat-Behandlung gelten Erosionen oder Ulzera der Haut und Schleimhäute. Hierzu zählen die ulzerierende Stomatitis und die erosive Umwandlung bereits vorhandener Dermatosen, z. B. einer Psoriasis vulgaris oder einer Stauungsdermatitis [20-22]. Das Auftreten von Erosionen gilt als klinischer Hinweis für eine unerkannte Überdosierung von Methotrexat oder für eine meist nicht berücksichtigte Interaktion mit anderen Arzneimitteln, vor allem mit Antibiotika oder nichtsteroidalen Antiphlogistika [23,24]. Für die toxische Pathogenese der Erosionen sprechen das gleichzeitige Auftreten toxischer Allgemeinsymptome, wie z. B. gastrointestinale Blutungen oder Panzytopenien, das Ausbleiben erneuter Erosionen bei reduzierter Methrotrexat-Therapie und histopathologische Veränderungen in Form dyskeratotischer Zellen oder kompletter Epidermisnekrosen $[25,26]$. Neben der diffusen Alopezie, der erosiven Umwandlung bestehender Dermatosen oder der Entwicklung primärer Ulzerationen werden auch palmoplantare Erytheme mit Desquamation als toxische Reaktion einer MethotrexatBehandlung angesehen. Diese nur selten beschriebene Nebenwirkung wurde bei Patienten mit Lymphomen oder Leukämien beobachtet, die eine hochdosierte Methotrexat-Therapie erhalten hatten [27]. Die Symptomatik entwickelte sich typischerweise wenige Stunden nach der ersten Methotrexat-Gabe und war in ihrer Ausprägung bei den nachfolgenden Behandlungszyklen von der dann gewählten Dosierung abhängig. Neben den toxischen Komplikationen sind bei Methotrexat auch verschiedene allergische Reaktionsformen bekannt geworden. Anaphylaktische Nebenwirkungen zeigten sich als generalisierter Pruritus, als urtikarielle Exantheme oder in Form ausgeprägter QuinckeÖdeme $[12,28,29]$. Die kutane Symptomatik war bei den betroffenen Patienten in der Regel mit kardiovaskulären Zeichen der Anaphylaxie und mit Bewusstseinsstörungen kombiniert. Die anaphylaktischen Reaktionen entwickelten sich stets erst nach wiederholten Methotrexat-Behandlungen, traten unmittelbar während der Medikamenten-Gabe auf und bildeten sich unter einer Behandlung mit Antihistaminika und Glukokortikoiden schnell zurück. In Einzelfällen konnte die Sensibilisierung durch Nachweis positiver Prick-Testungen belegt werden [29]. Weitere durch Methotrexat verursachte allergische Dermatosen sind makulopapulöse Arzneimittelexantheme, bei denen pathophysiologisch neben einer Typ IV-Sensibilisierung auch die Möglichkeit einer Hypersensitivitätsreaktion diskutiert wurde, sowie die Vasculitis allergica [30,31]. Während die bisher genannten kutanen Nebenwirkungen einer Methotrexat-Therapie durch toxische oder allergische Mechanismen ausgelöst werden, konnte die Pathogenese bei einer Reihe weiterer durch Methotrexat verursachter Dermatosen bisher nicht aufgeklärt werden. Hierzu zählen die toxische epidermale Nekrolyse, das Stevens-JohnsonSyndrom, die Reaktivierung UV-Licht-induzierter Erytheme, die Induktion von Rheumaknoten sowie die Entwicklung von Lymphomen und Pseudolymphomen [13 - 16,32 - 35]. Für die Bewertung der hier in der vorgestellten Kasuistik beschriebenen Arzneimittelreaktion sind die nach Methotrexat-Behandlung beobachteten toxischen epidermalen Nekrolysen und Stevens-Johnson-Syndrome von besonderer Bedeutung [13,33-35]. Unter dem Begriff der schweren blasenbildenden Hautreaktionen können die toxische epidermale Nekrolyse, das Stevens-JohnsonSyndrom, das Erythema exsudativum multiforme majus und das generalisierte bullöse fixe Arzneimittelexanthem zusammengefasst werden. Bei allen Unterschieden in der Schwere und im Verlauf dieser Dermatosen ist deren gemeinsame klinische Morphe gekennzeichnet durch umschriebene oder flächenhafte Erytheme, die mit einer unterschiedlich ausgeprägten Blasenbildung einhergehen [36]. In Übereinstimmung mit dieser gemeinsamen klinischen Morphologie sind auch die histopathologischen Veränderungen weitgehend identisch. Neben umschriebenen oder die gesamte Epidermis durchsetzenden nekrotischen Keratinozyten findet sich eine Vakuolisierung der Junktionszone bis hin zur subepidermalen Spaltbildung. Darüber hinaus kann in der Dermis ein superfizielles perivaskuläres lymphohistiozytäres Infiltrat beobachtet werden, das vereinzelt auch eosinophile Granulozyten enthält [37].

\section{Arzneimittelreaktion als lokale toxische epidermale Nekrolyse}

Die klinische Morphologie und die histopathologischen Veränderungen der hier vorgestellten Patientin stimmen mit den entsprechenden Befunden der schweren blasenbildenden Hautreaktionen überein. Im Gegensatz zu diesen Dermatosen traten die bullösen Erytheme bei unserer Patientin jedoch nur im Bereich der Methotrexat-Injektionen auf, so dass man bei fehlender Generalisation der Morphen und fehlender Paraklinik die bullöse Arzneimittelreaktion unserer Patientin auch als lokale toxische epidermale Nekrolyse auffassen kann. Die Pathogenese der schweren bullösen Arzneimittelreaktionen ist ungeklärt, wobei die klassischen allergischen Reaktionsformen nach Coombs und Gell oder direkte toxische Mechanismen als Erklärungsmodelle ausgeschlossen werden können. Bei der toxischen epidermalen Nekrolyse wird ein zytotoxisches Geschehen diskutiert, bei dem es unter dem Einfluss von Arzneimitteln zu einer Veränderung der Antigenstruktur der Keratinozyten kommen könnte [28]. Andere Überlegungen gehen davon aus, dass die Apoptose der Keratinozyten ausgelöst wird durch lytisch wirksame FAS-Ligandenmoleküle, die an FAS-Antigen auf der Keratinozytenoberfläche binden. Die vermutlich medikamentös induzierte verstärkte Expression von Ligandenmolekülen konnte bei Patienten mit einer toxischen epidermalen Nekrolyse nachgewiesen werden [39]. Die Pathogenese der von uns beobachteten bullösen Arzneimittelreaktion erklärt sich ebenfalls nicht durch allergische oder direkt toxische Mechanismen. Die blasigen Erytheme der Patientin zeigten sich erstmals 6 Monate nach Beginn der MethotrexatTherapie, was einerseits eine direkte toxische Reaktion ausschließt, andererseits als Hinweis für einen immunologischen Prozess gewertet werden kann. Die Latenz von 7-10 Tagen zwischen den Methotrexat-Injektionen und dem Auftreten der Mor- 
phen entspricht jedoch nicht den üblichen Zeitphasen der klassischen allergischen Reaktionsformen. Darüber hinaus zeigen Arzneimittelallergien, von wenigen Ausnahmen abgesehen, exanthematische Manifestationen, während bei unserer Patientin trotz monatelang fortgesetzter Methotrexat-Behandlung die Reaktionen nur an den Injektionsstellen auftraten. Auch die Ergebnisse der allergologischen Testungen und der histopathologischen Untersuchungen lassen sich mit keinen herkömmlichen pathogenetischen Konzepten vereinbaren. Die Prick-, Scratchund Epikutan-Testungen sowie die subkutanen Injektionen mit $1 \mathrm{mg}$ und $3 \mathrm{mg}$ Methotrexat verliefen unauffällig. Erst bei Injektion von $10 \mathrm{mg}$ Methotrexat konnte ein bullöses Erythem ausgelöst werden. Die erhobenen histopathologischen Befunde einer vakuolären Interface-Dermatitis waren wiederum typisch für eine schwere bullöse Arzneimittelreaktion. Gegen eine direkte toxische Reaktion, die aufgrund der kutanen Testungen und aufgrund der von der Patientin zusätzlich eingenommenen nichtsteroidalen Antiphlogistika zu diskutieren gewesen ist, sprachen im histopathologischen Bild das Fehlen von Veränderungen in der tiefen Dermis bei subkutaner Methotrexat-Injektion sowie das Fehlen von Nekrosezonen und neutrophilen Granulozyten, wie sie üblicherweise in der Umgebung von Nekrosen zu finden sind. In Übereinstimmung mit den kutanen Testungen bestand histopathologisch kein Anhalt für eine allergische Kontaktdermatitis, da in diesem Fall anstelle der vakuolären InterfaceDermatitis eine spongiotische Dermatitis zu erwarten gewesen wäre. Letztendlich bleibt die Pathogenese der hier beschriebenen bullösen Arzneimittelreaktion ungeklärt, wobei eine vergleichbare Beobachtung in der uns zur Verfügung stehenden Literatur nicht gefunden werden konnte.

\section{Literatur}

${ }^{1}$ Osieka R, Efferth T. Antineoplastische Substanzen. In: Schmoll H-J, Höffgen K, Possinger K (Hrsg). Kompendium Internistische Onkologie Teil 1. Berlin: Springer, 1999

2 Kremer JM. Methotrexate update. Scand J Rheumatol 1996; 25: 341 344

${ }^{3}$ Gollnick H, Bonnekoh B. Psoriasis-Pathogenese, Klinik und Therapie. Bremen: UNI-MED, 2001

${ }^{4}$ Mahrle G. Die systemische Therapie der Psoriasis. HuG 1996; 71: 455 464

${ }^{5}$ Kind P. Methotrexat. In: Plewig G, Wolff H (Hrsg). Fortschritte der praktischen Dermatologie und Venerologie 1998. Berlin: Springer, 1999

${ }^{6}$ Van Dooren-Greebe RJ, Kuijpers ALA, Mulder J et al. Eine Neubewertung des Methotrexats: Ergebnisse der Langzeitbehandlung von Psoriasis. HuG 1994; 69: 136- 142

${ }^{7}$ Zachariae H. Methotrexte side-effects. Br J Dermatol 1990; 122 (Suppl 36): $127-133$

${ }^{8}$ Roenigk HH, Auerbach R, Maibach HI et al. Methotrexate in psoriasis: revised guidelines. J Am Acad Dermatol 1988; 19: 145-156

${ }^{9}$ Zachariae H, Sogaard H. Methotrexate-induced liver cirrhosis. A follow-up. Dermatologica 1987; 175: 178- 182

10 al-Awadhi A, Dale P, McKendry RJ. Pancytopenia associated with low dose methotrexate therapy. A regional survey. J Rheumatol 1993; 20: $1121-1125$

11 From E. Methotrexate pneumonitis in a psoriatic. Br J Dermatol 1975; 93: $107-110$

12 Gluck-Kuyt I, Irwin LE. Anaphylactic reaction to high-dose methotrexate. Cancer Treat Rep 1979; 63: 797 - 798
${ }^{13}$ Hani N, Casper C, Groth W et al. Stevens-Johnson syndrome-like exanthema secondary to methotrexate histologically simulating acute graff-versus-host disease. Eur J Dermatol 2000; 10: 548 - 550

${ }^{14}$ Kerstens PJ, Boerbooms AMT, Jeurissen M et al. Accelerated nodulosis during low dose methotrexate therapy for rheumatoid arthritis. An analysis of ten cases. J Rheumatol 1992; 19: 867-871

${ }^{15}$ Korossy KS, Hood AF. Methotrexate reactivation of sunburn reaction. Arch Dermatol 1981; 117: 310-311

${ }^{16}$ Viraben R, Brousse P, Lamant L. Reversible cutaneous lymphoma occuring during methotrexate therapy. Br J Dermatol 1996; 135: 116 118

${ }^{17}$ Allegra CF, Fine RL, Drake JC et al. The effect of methotrexate on intracellular folate pools in human MCF7 breast cancer cells: evidence for direct inhibition of purine synthesis. J Biol Chem 1986; 261: 6478 6485

${ }^{18}$ Evans WE, Christensen ML. Drug interactions with methotrexate. J Rheumatol 1985; 12 (Suppl 12): 15 - 20

${ }^{19}$ Sonnveld P, Schultze FW, Nooter K et al. Pharmacokinetics of methotrexate and 7-hydroxymethotrexate in plasma and bone marrow of children receiving low-dose oral methotrexate. Cancer Chemother Pharmacol 1986; 18: 111 - 116

${ }^{20}$ Dreizen S. Stomatotoxic manifestations of cancer chemotherapy. J Prosthet Dent 1978; 40: 650-655

${ }^{21}$ Kaplan DL, Olsen EA. Erosion of psoriatic plaques after chronic methotrexate administration. Int J Dermatol 1988; 27: 59-62

${ }^{22}$ Lawrence CM, Dahl MG. Two patterns of skin ulceration induced by methotrexate in patients with psoriasis. J Am Acad Dermatol 1984; 11: $1059-1065$

${ }^{23}$ Bauer J, Fartasch M, Schuler G et al. Ulzerierende Stomatitis als klinischer Hinweis auf eine unerkannte Überdosierung von Methotrexat. Hautarzt 1999; 50: 670-673

${ }^{24}$ Pearce H, Braunstein Wilson B. Erosion of psoriatic plaques: An early sign of methotrexate toxicity. J Am Acad Dermatol 1996; 35: 835 - 838

${ }^{25}$ Baker H. Intermittent high dose oral methotrexate therapy in psoriasis. Br J Dermatol 1970; 82: 65 - 69

${ }^{26}$ Reed KM, Sober AJ. Methotrexate-induced necrolysis. J Am Acad Dermatol 1983; 8: 677-679

${ }^{27}$ Doyle LA, Berg C, Bottino G et al. Erythema and desquamation after high-dose methotrexate. Ann Intern Med 1983; 98: 611 - 612

${ }^{28}$ Goldberg NH, Romolo JL, Austin EH et al. Anaphylactoid type reactions in two patients receiving high dose intravenous methotrexate. Cancer 1978; 41: $52-55$

${ }^{29}$ Paradis L, Des Roches A, Paradis J et al. Methotrexate allergy. J Allergy Clin Immunol 1995; 95: 289

${ }^{30}$ Marks CR, Willkens RF, Wilske KR et al. Small-vessel vasculitis and methotrexate. Ann Intern Med 1984; 100: 916

${ }^{31}$ Sitki C, William D, Edward C et al. Bone marrow aplasia and severe skin rash after a single low dose of methotrexate. Anti-Cancer Drugs 1995; 6: $154-157$

${ }^{32}$ Cobeta-Garcia JC, Ruiz-Jimeno MT, Fontova-Garrofe R. Non-Hodgkin's lymphoma, rheumatoid arthritis and methotrexate. J Rheumatol 1993; 20: 200-202

33 Cuthbert RJ, Craig JIO, Ludlam CA. Stevens-Johnson syndrome associated with methotrexate treatment for Non Hodgkin's lymphoma. Ulster Med J 1993; 62: 95 - 97

${ }^{34}$ Moe PJ, Seip M. High dose methotrexate in acute lymphocytic leukaemia in childhood. Acta Paediat Scand 1978; 67: 265-268

${ }^{35}$ Yang $\mathrm{CH}$, Yang LJ, Jaing TH et al. Toxic epidermal necrolysis following combination of methotrexate and trimethoprim-sulfamethoxazole. Int J Dermatol 2000; 39: 621 - 623

${ }^{36}$ Rzany B, Mockenhaupt M, Baur S et al. Schwere Hautreaktionen. Hautarzt 1993; 44: 549-556

37 Schöpf E, Mockenhaupt M. Arneimittelbedingte schwere Hautreaktionen, Stevens-Johnson-Syndrom und Toxische epidermale Nekrolyse. In: Tebbe B, Goerdt S, Orfanos CE (Hrsg). Dermatologie-Heutiger Stand. Stuttgart: Thieme, 1995

${ }^{38}$ Heng CY, Allen SG. Efficacy of cyclophosphamide in toxic epidermal necrolysis. J Am Acad Dermatol 1991; 25: 778 - 786

${ }^{39}$ Viard I, Wehrli P, Bullani R et al. Inhibiton of toxic epidermal necrolysis by blockade of CD95 with human intravenous immunglobulin. Science 1998; 282: 490-493 